\title{
A Multi-Input Cross-Connected Charge Pump for Mobile Applications
}

\author{
Kei Eguchi ${ }^{a}$, Farzin Asadi ${ }^{b}$, Takaaki Ishibashic, Ichirou Oota ${ }^{c}$ \\ aDepartment of Information Electronics, Fukuoka Institute of Technology, \\ 3-30-1 Wajirohigashi, Higashi-ku, Fukuoka, 811-0295 Japan \\ ${ }^{b}$ Mechatronics Engineering Department, Kocaeli University, \\ Umuttepe Yerleşkesi 41380, Kocaeli, Turkey \\ 'Department of Electronics Engineering and Computer Science, \\ National Institute of Technology, Kumamoto College \\ 2659-2 Suya, Koushi-shi, Kumamoto, 861-1102 Japan \\ *Corresponding Author: eguti@fit.ac.jp
}

\begin{abstract}
To develop efficient mobile application systems, a multiinput cross-connected charge pump is proposed in this paper. By combing ambient energy and battery energy, the proposed charge pump achieves a long working-life of the battery. Furthermore, unlike conventional charge pumps, the converter blocks are cross-connected in the proposed charge pump. Therefore, the proposed charge pump can realize smaller ripple noise than the traditional charge pump, because the output voltage is provided to an output load at every clock cycle. To clarify the characteristics of the proposed charge pump, we conducted theoretical analysis and simulation program with integrated circuit emphasis (SPICE) simulations. The theoretical analysis revealed the characteristics such as power efficiency and output voltage theoretically. Furthermore, SPICE simulation showed that the proposed charge pump can achieve efficient power efficiency and circuit speed.
\end{abstract}

Keywords: charge pump circuits, energy harvesting, inductor-less converters, power converters.

\section{Introduction}

Recently, energy harvesting technology is receiving much attention in the field of mobile applications, such as wireless sensor networks, wearable electronics, etc. To utilize ambient energy, a switching converter is usually used to convert small energy harvested by thermoelectric generators (TEGs), photovoltaics, piezoelectric devices, and so on. Among others, an inductor-less switching converter such as a charge pump ${ }^{(1)-(5)}$ is the most promising power converters, because it can realize no flux of magnetic induction and small size owing to inductor-less topology.

In past studies, many researchers have tackled to develop an efficient charge pump by utilizing ambient energy. For example, Doms et al. ${ }^{(1)}$ and Yun et al. ${ }^{(2)}$ proposed a charge pump for TEGs. By converting thermal energy produced by TEGs, Doms and Yun's charge pump can offer a high stepped-up voltage without batteries. However, it generates the output voltage only in a half clock cycle. Therefore, it requires a big output capacitor to suppress output ripple. To stabilize a supply voltage of energy harvesting systems, Eguchi et al. ${ }^{(3),(4)}$ suggested a multipleinput charge pump by combining a clean energy source and a battery source. However, Eguchi's charge pump suffers from the same problem as that of Doms and Yun's charge pump. Following these studies, in order to improve output ripple and power efficiency, a split-merge charge pump was proposed by Wang et al. ${ }^{(5)}$. However, the circuit control of Wang's charge pump is complex, because it requires multiphase clock pulses. Furthermore, due to parallel-connected structure, the circuit size of Wang's charge pump is much larger than that of previous charge pumps ${ }^{(1)-(4)}$.

In this paper, a multi-input cross-connected charge pump is proposed for mobile applications. By combing ambient energy and battery energy, the proposed charge pump achieves a long working-life of the battery. Furthermore, unlike conventional charge pumps, the proposed charge pump has a cross-connected structure. Therefore, the proposed charge pump can realize smaller ripple noise than the traditional charge pump, because the output voltage is 
provided to an output load at every clock cycle.

Concerning power efficiency and output voltage, the characteristics of the proposed charge pump are analyzed theoretically. Furthermore, through simulation program with integrated circuit emphasis (SPICE) simulations, the effectiveness of the proposed charge pump is verified by comparing the characteristics of the proposed charge pump and the traditional charge pump.

The rest of this paper is as follows: In section 2, the circuit topologies of the proposed charge pump and the traditional charge pump are explained in detail. In section 3 , theoretical analysis is performed to reveal the characteristics of the proposed charge pump. Section 4 shows the SPICE simulated results to demonstrate the effectiveness of the proposed charge pump. Lastly, section 6 summarizes the results of this study and future works.

\section{Circuit Configuration}

\subsection{Conventional Charge Pump}

Figure 1 shows the circuit configuration of the conventional charge pump ${ }^{(1),(2)}$, where $S_{1}$ and $S_{2}$ are transistor switches controlled by non-overlapped two-phase clock pulses. The output voltage of the conventional charge pump is expressed by

$$
V_{\text {out }}=(N+1) V_{\text {in }} \text {, }
$$

where the parameter $N$ denotes the number of stages. Concretely, in the case of figure 1 , the parameter $N$ is 3 . According to the number of stages, $N$, the step-up gain of the conventional charge pump increases linearly.

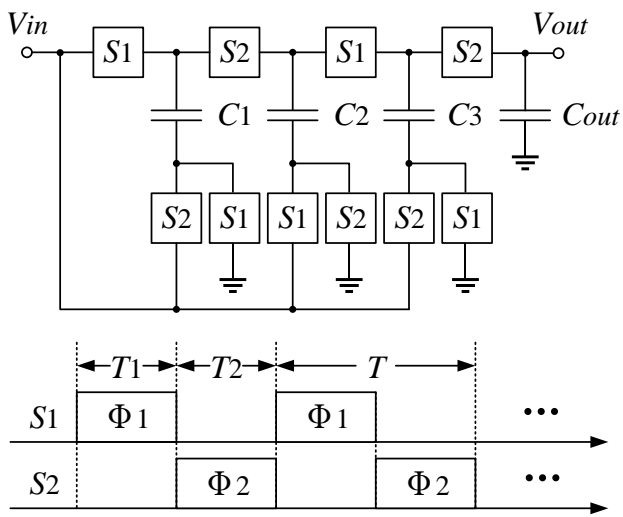

Fig. 1 Circuit configuration of the conventional charge pump.
However, as figure 1 shows, the conventional charge pump generates an output voltage only at a half clock cycle. Therefore, in order to realize small ripple noise, the conventional charge pump requires a big output capacitor $C_{\text {out }}$. Of course, by using a multistep split-merge charge transfer operation ${ }^{(5)}$, the conventional charge pump can improve the output ripple. However, due to multi-phase clock pulses and parallel structure, the conventional charge pump $^{(5)}$ suffers from complex circuit operation and large circuit size

\subsection{Proposed Charge Pump}

Figure 2 illustrates the circuit configuration of the proposed charge pump. As figure 2 shows, the proposed charge pump has two inputs: a battery input and an ambient energy input. By utilizing ambient energy, the proposed charge pump alleviates power consumption from the battery. In figure 2 , the selection circuit is controlled according to the generation situation of the ambient energy source. Of course, there is no need to use the selection circuit if the voltage provided by the ambient energy source is stable. Furthermore, by using the cross-connected structure, the output voltage is provided to an output load at every clock cycle, because the converter block-1 and 2 are operated at opposite polarity.

The output voltage of the proposed charge pump is expressed as

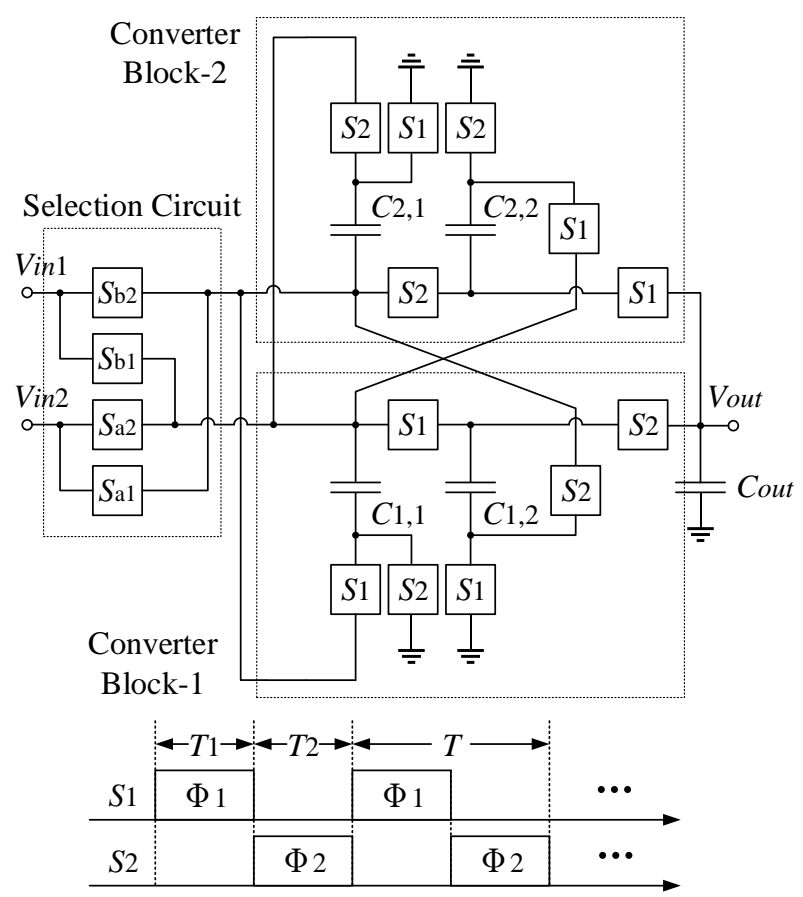

Fig. 2 Circuit configuration of the proposed charge pump with $4 \times$ step-up gain 


$$
V_{\text {out }}=\left\{\begin{array}{ccc}
N\left(V_{i n 1}+V_{i n 2}\right) & \text { if } & S_{a 2} \text { and } S_{b 2} \text { are ON } \\
2 N V_{i n 1} & \text { if } & S_{b 1} \text { and } S_{b 2} \text { are ON } \\
2 N V_{i n 2} & \text { if } & S_{a 1} \text { and } S_{a 2} \text { are ON }
\end{array}\right.
$$

As (2) shows, according to the generation situation of the ambient energy source, the proposed charge pump controls the selection circuit to extend the lifetime of the battery. For above-mentioned reasons, the proposed charge pump can reduce not only long working-lift but also small ripple noise.

\section{Theoretical Analysis}

Concerning the proposed charge pump, the circuit characteristics, such as power efficiency and output voltage, are analyzed theoretically. In the theoretical analysis, the four-terminal equivalent $\operatorname{model}^{(3),(4)}$ shown in figure 3 is assumed to obtain the maximum power efficiency and the maximum output voltage. In figure 3 , the parameter $R$ denotes the conversion ratio of an ideal transformer and the parameter $R_{S C}$ denotes an internal resistance which is called the SC resistance. By deriving these parameters $R$ and $R_{S C}$ theoretically, we express the proposed charge pump as the four-terminal equivalent circuit. By utilizing the equivalent circuit, mathematical formulas are obtained to estimate the maximum power efficiency and the maximum output voltage.

In the following subsections, the characteristics of the proposed charge pump will be compared with that of the conventional charge pump theoretically.

In a steady state, the instantaneous equivalent circuits of the conventional charge pump are illustrated by figure 4 , where $R_{\text {on }}$ denotes an on-resistance of the transistor switch. In figure 4 , the differential value of electric charges $\Delta q_{T 1}^{i, j}$ and $\Delta q_{T 2}{ }^{i, j}$ in $C_{k}((i=1,2)$ and $(j=1,2))$ satisfies the following condition:

$$
\Delta q_{T_{1}}^{i, j}+\Delta q_{T_{2}}^{i, j}=0
$$

In (3), $\Delta q_{T k^{i, j}}(i=1,2)$ denotes the electric charge of the $k$-th capacitor $C_{j}(j=1,2)$ in State- $T_{k}(k=1,2)$. The intervals $T_{1}$ and $T_{2}$ satisfy the following conditions:

$$
T=T_{1}+T_{2} \text { and } T_{1}=T_{2}=\frac{T}{2} .
$$

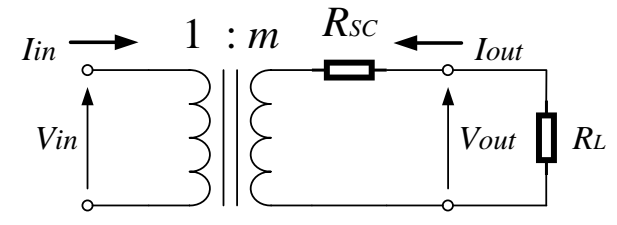

Fig. 3 Four-terminal equivalent model.

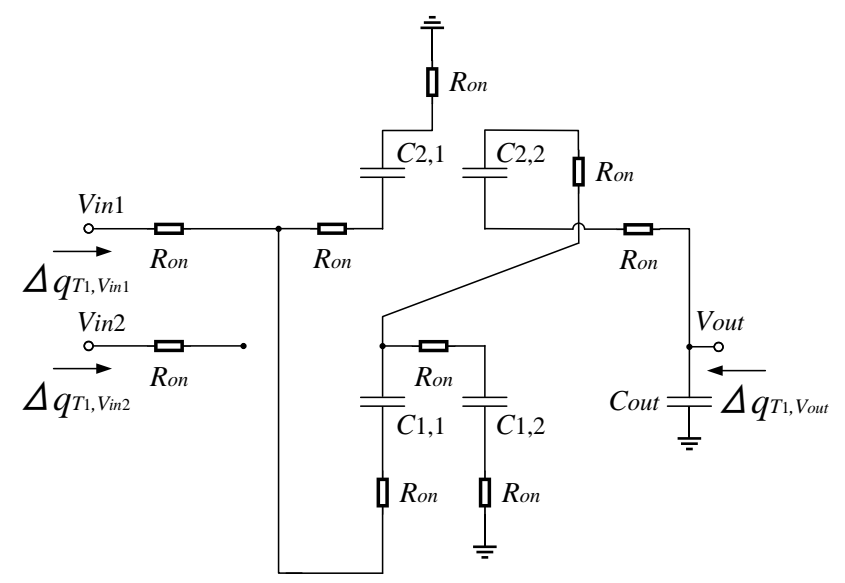

(a)

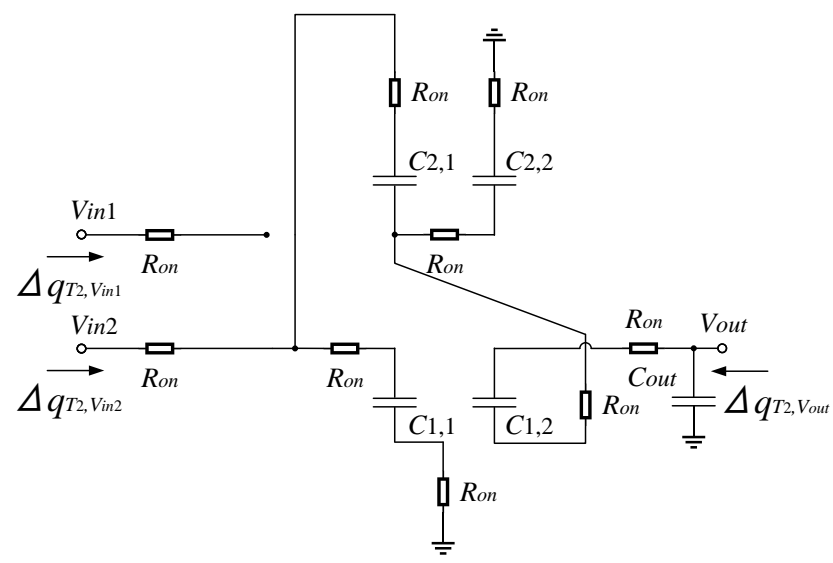

(b)

Fig. 4 Instantaneous equivalent circuits of the conventional charge pump: (a) state- $T_{1}$ and (b) state- $T_{2}$.

In State- $T_{1}$, the differential values of electric charges in the input and the output, $\Delta q_{T 1, v i n 1}, \Delta q_{T 1, v i n 2}$, and $\Delta q_{T 1, v o u t}$, are expressed as

$$
\begin{aligned}
& \Delta q_{T_{1}, v_{\text {in } 1}}=\Delta q_{T_{1}}^{2,1}-\Delta q_{T_{1}}^{1,1}, \\
& \Delta q_{T_{1}, v_{\text {in } 2}}=0 \\
& \Delta q_{T_{1}, v_{\text {out }}}=\Delta q_{T_{1}}^{2,2}+\Delta q_{T_{1}}^{\text {out }}
\end{aligned}
$$$$
\text { and }
$$ 
On the other hand, in State- $T_{2}$, the differential values of electric charges, $\Delta q_{T 2, v i n}, \Delta q_{T 2, v i n 2}$, and $\Delta q_{T 2, v o u t}$, are expressed as

$$
\begin{aligned}
\Delta q_{T_{2}, v_{\text {in } 1}} & =0, \\
\Delta q_{T_{2}, v_{\text {in } 2}} & =\Delta q_{T_{2}}^{1,1}-\Delta q_{T_{2}}^{2,1}, \\
\text { and } \quad \Delta q_{T_{2}, v_{\text {out }}} & =\Delta q_{T_{2}}^{1,2}+\Delta q_{T_{2}}^{\text {out }}
\end{aligned}
$$

Furthermore, the following conditions are satisfied:

$$
\begin{aligned}
& \Delta q_{T_{1}}^{2,2}=\Delta q_{T_{1}}^{1,1}+\Delta q_{T_{1}}^{1,2}, \\
\text { and } \quad \Delta q_{T_{2}}^{1,2} & =\Delta q_{T_{2}}^{2,1}+\Delta q_{T_{2}}^{2,2}
\end{aligned}
$$

In a steady state, the overall change of the input/output (I/O) currents is zero. Therefore, by using (5) and (6), the I/O currents $I_{\text {in } 1}, I_{\text {in } 2}$, and $I_{\text {out }}$ can be expressed as

$$
\begin{aligned}
I_{\text {in } 1} & =\frac{\Delta q_{v_{\text {in } 1}}}{T}=\frac{\Delta q_{T_{1}, v_{\text {in } 1}}+\Delta q_{T_{2}, v_{\text {in } 1}}}{T}, \\
\text { and } \quad I_{\text {in } 2} & =\frac{\Delta q_{v_{\text {in } 2}}}{T}=\frac{\Delta q_{T_{1}, v_{\text {in } 2}}+\Delta q_{T_{2}, v_{\text {in } 2}}}{T}, \\
& I_{\text {out }}= \\
& =\frac{\Delta q_{v_{\text {out }}}}{T}=\frac{\Delta q_{T_{1}, v_{\text {out }}}+\Delta q_{T_{2}, v_{\text {out }}}}{T}
\end{aligned}
$$

Where, $\Delta q_{v i n}, \Delta q_{v i n 2}$, and $\Delta q_{v o u t}$ are electric charges in $V_{\text {in } 1}$, $V_{\text {in } 2}$, and $V_{\text {out }}$, respectively. By substituting (3) - (7) into (8), the relation between $\mathrm{I} / \mathrm{O}$ currents can be derived as

$$
I_{\text {in } 1}+I_{\text {in } 2}=-4 I_{\text {out }},
$$

where

$$
I_{i n 1}=I_{i n 2}=-2 I_{o u t},
$$$$
\text { and } \quad \Delta q_{v_{\text {in } 1}}=\Delta q_{v_{\text {in } 2}}=-2 \Delta q_{v_{\text {out }}}
$$

Therefore, we have the parameter $R$ in figure 3 as $R=4$.

Next, in order to obtain the parameter $R_{S C}$, let us consider the consumed energy in one period. In figure 4 , energy is consumed by the on-resistance $R_{o n}$. Therefore, the consumed energy in State- $T_{1}$ is expressed as

$$
\begin{aligned}
W_{T_{1}}= & \frac{\left(\Delta q_{T_{1}}^{2,1}\right)^{2}}{T_{1}} 2 R_{o n}+\frac{\left(\Delta q_{T_{1}}^{1,1}\right)^{2}}{T_{1}} R_{o n}+\frac{\left(\Delta q_{T_{1}}^{1,2}\right)^{2}}{T_{1}} 2 R_{o n} \\
& +\frac{\left(\Delta q_{T_{1}}^{2,2}\right)^{2}}{T_{1}} 2 R_{o n}+\frac{\left(\Delta q_{T_{1}}^{1,1}-\Delta q_{T_{1}}^{2,1}\right)^{2}}{T_{1}} R_{o n},
\end{aligned}
$$

Here, as figure 4 shows, the proposed charge pump has a symmetrical structure. Therefore, the total consumed energy in one period is expressed as

$$
W_{T}=2 W_{T_{1}}=\frac{\left(\Delta q_{V_{\text {out }}}\right)^{2}}{T} 32 R_{\text {on }} .
$$

Here, the consumed energy of figure 3 can be defined by

$$
W_{T}=\frac{\left(\Delta q_{V_{\text {out }}}\right)^{2}}{T} R_{S C}
$$

Therefore, the parameter $R_{S C}$ in figure 3 can be derived as $R_{S C}=32 R_{o n}$. Finally, by combining the parameters $R$ and $R_{S C}$, the equivalent circuit can be expressed by figure 5 . Therefore, from figure 5 , we have the equivalent circuit of the conventional charge pump by the following K-matrix:

$$
\left[\begin{array}{c}
V_{\text {in }} \\
I_{\text {in }}
\end{array}\right]=\left[\begin{array}{cc}
1 / 4 & 0 \\
0 & 4
\end{array}\right]\left[\begin{array}{cc}
1 & 32 R_{\text {on }} \\
0 & 1
\end{array}\right]\left[\begin{array}{c}
V_{\text {out }} \\
-I_{\text {out }}
\end{array}\right],
$$

where $V_{i n}=V_{i n 1}=V_{i n 2}$. From (14), the power efficiency $\eta$ and the output voltage $V_{\text {out }}$ are obtained as follows:

$$
\begin{array}{cc} 
& \eta=\frac{R_{L}}{32 R_{\text {on }}+R_{L}} \\
\text { and } \quad V_{\text {out }}=\left(\frac{R_{L}}{32 R_{\text {on }}+R_{L}}\right) \times 4 V_{\text {in }}
\end{array}
$$

As (15) shows, the characteristics of the charge pump depends on the parameter $R_{S C}$. Of course, the proposed charge pump does not require the selection circuit if the ambient energy source is stable. In this case, the proposed charge pump can be rewritten by figure 5 , because there is not use to control the selection circuit. In the case of figure 5 , the power efficiency $\eta$ and the output voltage $V_{\text {out }}$ are expressed by 


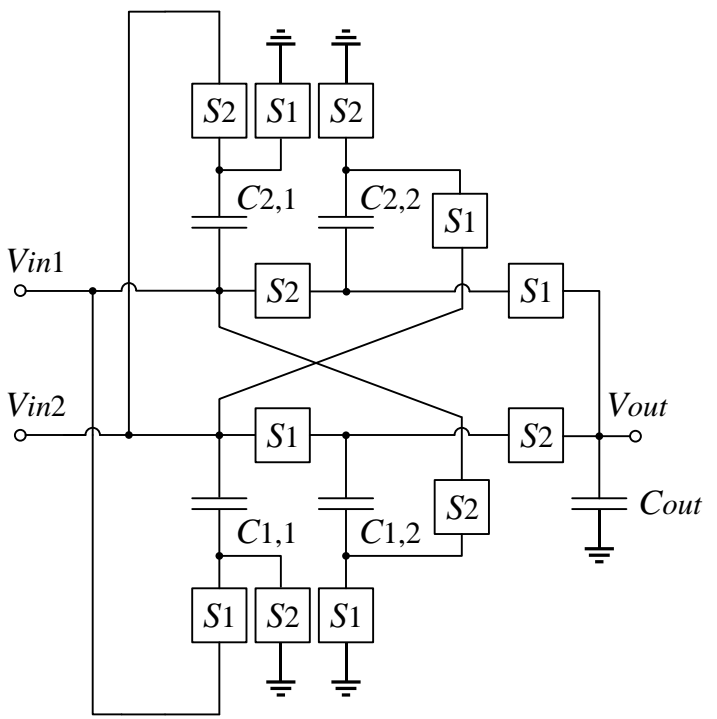

Fig. 5 Circuit configuration of the proposed charge pump without selection circuit

$$
\begin{aligned}
& \eta=\frac{R_{L}}{16 R_{\text {on }}+R_{L}} \\
& \text { and } \quad V_{\text {out }}=\left(\frac{R_{L}}{16 R_{\text {on }}+R_{L}}\right) \times 4 V_{\text {in }}
\end{aligned}
$$

\section{Comparison}

Table 1 demonstrates the comparison of $\eta_{\max }$ and $V_{\max }$ between the proposed charge pump and the conventional charge pump. As Table 1 shows, the proposed charge pump without the selection circuit is higher power efficiency and output voltage than the conventional charge pump. On the other hand, the power efficiency of the proposed charge pump with the selection circuit is lower than that of the conventional charge pump. In other words, if the voltage of the ambient energy source is stable, the proposed charge pump can achieve higher power efficiency and output voltage than the conventional charge pump.

Table 2 shows the comparison of the number of circuit components between the proposed charge pump and the conventional charge pump. As Table 2 shows, the number of circuit components of the conventional charge pump is smaller than that of the proposed converter. However, the conventional charge pump requires a big output capacitor to reduce ripple nose, because the conventional charge pump generates the output voltage only at a half clock cycle. It is known that the occupational chip area of capacitors is much bigger than that of transistor switches. For this reason, the proposed charge pump can achieve high response speed and
Table 1. Comparison of characteristics

\begin{tabular}{|c|c|c|}
\hline $\begin{array}{c}\text { Power } \\
\text { Pump with } \\
\text { selection circuit }\end{array}$ & $\frac{R_{L}}{32 R_{o n}+R_{L}}$ & $\left(\frac{R_{L}}{32 R_{o n}+R_{L}}\right) \times 4 V_{i n}$ \\
\hline $\begin{array}{c}\text { Proposed Charge } \\
\text { Pump without } \\
\text { selection circuit }\end{array}$ & $\frac{R_{L}}{16 R_{o n}+R_{L}}$ & $\left(\frac{R_{L}}{16 R_{o n}+R_{L}}\right) \times 4 V_{\text {in }}$ \\
\hline $\begin{array}{c}\text { Conventional } \\
\text { Charge Pump }\end{array}$ & $\frac{R_{L}}{20 R_{o n}+R_{L}}$ & $\left(\frac{R_{L}}{20 R_{o n}+R_{L}}\right) \times 4 V_{i n}$ \\
\hline
\end{tabular}

Table 2. Comparison of the number of circuit components

\begin{tabular}{|c|c|c|}
\hline & $\begin{array}{c}\text { Number of } \\
\text { Switches }\end{array}$ & $\begin{array}{c}\text { Number of } \\
\text { Capacitors }\end{array}$ \\
\hline $\begin{array}{c}\text { Proposed Charge } \\
\text { Pump with } \\
\text { selection circuit }\end{array}$ & 18 & 5 \\
\hline $\begin{array}{c}\text { Proposed Charge } \\
\text { Pump without } \\
\text { selection circuit }\end{array}$ & 14 & 5 \\
\hline $\begin{array}{c}\text { Conventional } \\
\text { Charge Pump }\end{array}$ & 10 & 4 \\
\hline
\end{tabular}

small occupational chip area though the number of circuit components of the proposed charge pump is larger than that of the conventional charge pump.

\section{Simulation}

To clarify the characteristics of the proposed charge pump, SPICE simulators were performed under the following conditions $^{(6)}$ : the input voltage $V_{i n 1}=V_{i n 2}=400 \mathrm{mV}$ (i.e. both inputs are assumed as ambient energy sources.), the onresistance of transistor switches $R_{o n}=1 \Omega$, the capacitance of the proposed charge pump $C_{1}=\ldots=C_{4}=C_{\text {out }}=200 \mathrm{pF}$, the capacitance of the conventional charge pump $C_{1}=\ldots=C_{3}=$ $200 \mathrm{pF}$ and $C_{\text {out }}=5 \mathrm{nF}$, the operating frequency $f=1 \mathrm{MHz}$, and the period of clock pulse $T=10 \mu \mathrm{s}$ and $T_{1}=T_{2}=5 \mu \mathrm{s}$.

The comparison of the simulated output voltage is demonstrated in figure 6. In the simulation conditions of figure 6 , the ideal output voltage is $1.6 \mathrm{~V}$. As figure 6 shows, owing to the cross-connected structure, the proposed charge pump can provide higher output voltage than the conventional charge pump. Concretely, about $80 \mathrm{mV}$ output voltage was improved by the proposed charge pump when the output power is $30 \mu \mathrm{W}$. 
The comparison of the simulated power efficiency is shown in figure 7. As figure 7 shows, the proposed charge pump can achieve higher power efficiency than the conventional charge pump when the output power is larger than about $20 \mu \mathrm{W}$. About 5\% power efficiency was improved by the proposed charge pump when the output power is 30 $\mu \mathrm{W}$.

The comparison of the simulated response speed is shown in figure 8 , where the output load was set to $10 \mathrm{k} \Omega$. As figure 8 shows, the proposed charge pump is faster than the conventional charge pump, because a big output capacitor is not necessary for the proposed charge pump. When the output load is $10 \mathrm{k} \Omega$, the proposed charge pump improved the settling time about $150 \mu \mathrm{s}$.

Of course, by using a big output capacitor, the conventional charge pump can improve the characteristics such as output voltage, power efficiency, and response speed. However, the conventional charge pump suffers from the occupational chip area. Concretely, it is difficult to implement a nano-order size capacitor into an IC chip.

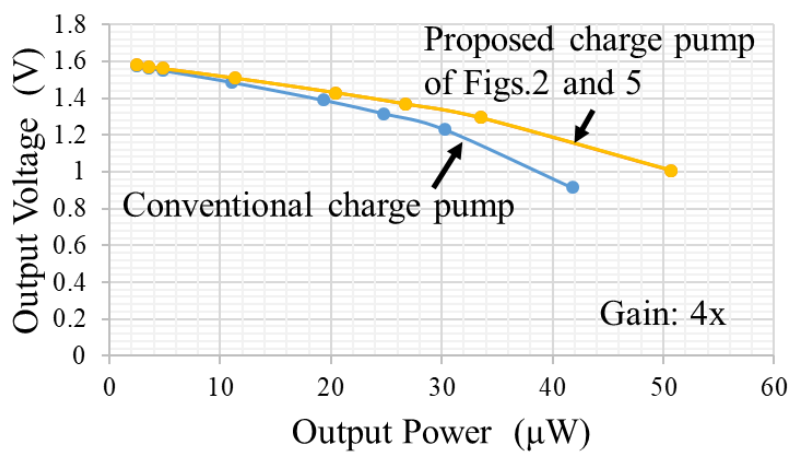

Fig. 6 Simulated output voltage as a function of the output power.

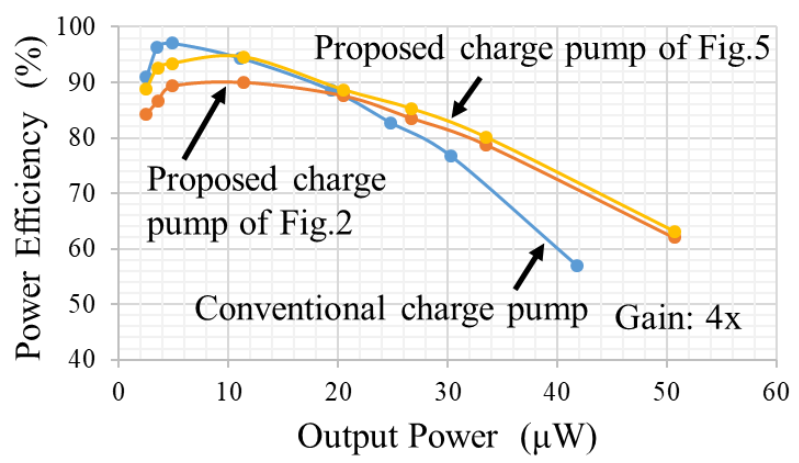

Fig. 7 Simulated power efficiency as a function of the output power.

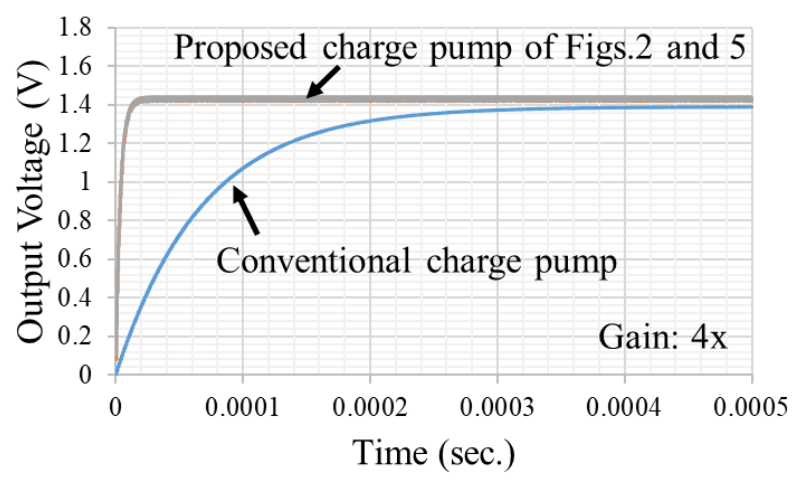

Fig. 8 Simulated output voltages as a function of the time.

\section{Conclusions}

To develop efficient mobile application systems, a multiinput cross-connected charge pump has been proposed in this paper. By combing ambient energy and battery energy, the proposed charge pump achieves a long working-life of the battery. Furthermore, owing to the cross-connected structure, the proposed charge pump can realize smaller ripple noise than the traditional charge pump.

The results of theoretical analysis and SPICE simulations demonstrated the following results: 1 . When the output power is $30 \mu \mathrm{W}$, the proposed $4 \times$ step-up charge pump improved more than $5 \%$ power efficiency and $80 \mathrm{mv}$ output voltage from the conventional charge pump and 2 . The settling time of the proposed charge pump is about $150 \mu \mathrm{s}$ faster than that of the conventional charge pump when the output load is $10 \mathrm{k} \Omega$.

In a future study, we are going to implement the proposed charge pump into an IC chip.

\section{References}

(1) Inge Doms, Patrick Merken, Chris Van Hoof, and Robert P. Mertens: "Capacitive power management circuit for micropower thermoelectric generators with a $1.4 \mu \mathrm{A}$ controller", IEEE, Solid-State Circuits, Vol. 44, No. 10, pp. 2824-2833, 2009.

(2) Goh Siew Yun, Kok Swee Leong, and Mohd Fauzi Bin Abdul Rahman: "Development of charge pump circuit using multivibrator for thermoelectric generator", Proc. 2016 IEEE International Conference on Power and Energy (PECon), pp. 434-438, 2016

(3) Kei Eguchi, Tatsuya Sugimura, Sawai Pongswatd, Kitti Tirasesth, and Hirofumi Sasaki: "Design of a multipleinput parallel SC DC-DC converter and its efficiency estimation method", ICIC Express Letters, Vol. 3, No. 3, 
pp. 531-536, 2009.

(4) Kei Eguchi, Kuniaki Fujimoto, and Hirofumi Sasaki: “A hybrid input charge-pump using micropower thermoelectric generators", IEEJ Transactions on Electrical and Electronic Engineering, Vol. 7, No. 4, pp. 415-422, 2012.

(5) Yu Wang, Na Yan, Hao Min, and C. -J. Richard Shi: “A High-Efficiency Split-Merge Charge Pump for Solar Energy Harvesting", IEEE Transactions on Circuits and Systems II: Express Briefs, Vol. 64, No. 5, pp. 545-549, 2017.

(6) Yuanfei Wang, Ping Luo, Xinyi Zheng, and Bo Zhang: "A $0.3 \mathrm{~V}-1.2 \mathrm{~V}$ ultra-low input voltage, reconfigurable switched-capacitor DC-DC converter for energy harvesting system", Proc. 2016 13th IEEE International Conference on Solid-State and Integrated Circuit Technology (ICSICT), pp. 1333-1335, 2016. 